\title{
Indoor Wideband Time/Angle of Arrival Multipath Propagation Results
}

\author{
Quentin Spencer, Michael Rice, Brian Jeffs, and Michael Jensen \\ Department of Electrical \& Computer Engineering \\ Brigham Young University \\ Provo, Utah 84602
}

\begin{abstract}
Most current indoor propagation experiments measure the time of arrival of characteristics of multipath reflections without regard to angle of arrival. Because of the increasing number of systems that are used indoors and which use multiple antenna systems to combat multipath interference, a need exists for indoor propagation data which takes angle of arrival into account. In this paper, a system is described which was used to collect simultaneous time and angle of arrival data in two indoor environments. A total of 65 data sets were taken in two buildings of different construction. The data confirmed the temporal model proposed by Saleh and Valenzuela [1], and showed a unique clustering pattern in angle, which consisted of clusters uniformly distributed in angle, with members of the clusters following a Laplacian distribution.
\end{abstract}

\section{INTRODUCTION}

Radio has recently become an increasingly viable option for indoor communications applications. The availability of new, higher frequency bands has made wireless an attractive option for high bandwidth digital communications in applications such as local area networks. Wireless networks can be advantageous in situations where portability is desirable, or where installation of wiring is undesirable or impractical.

Multipath interference, or interference due to the reception of multiple copies of a signal due to reflections, is known to be a problem in many outdoor communication channels. As a result, much data has been collected in outdoor environments to characterize various multipath scenarios. However, multipath is also a problem in indoor environments, but has characteristics distinct from outdoor multipath because of the presence of walls and large objects which present very different reflection mechanisms. The increasing use of wireless in indoor applications demands greater study of the indoor multipath environment.

Several other researchers have already collected various types of data on indoor multipath propagation. The foundation for much of today's work was the paper by Turin, et al [2], which was a study of outdoor multipath propagation in an urban environment. This work eventually led to the paper by Saleh and Valenzuela, an important early reference on indoor multipath propagation. Their work consisted of collecting temporal data on indoor propagation, from which they proposed a time domain model for indoor propagation. Their model is discussed in the paper by Spencer et al [3].

Until very recently, nearly all of the work in indoor multipath propagation dealt with time domain data, but did not include any data on the angle of arrival. Knowledge of the angle associated with a multipath arrival is important because of the increasing use of multiple antenna systems in communications applications. Signals from multiple antennas can be exploited by using (for example) diversity combining, phased array beamforming, or adaptive array processing algorithms to overcome the effects of multipath. Additionally, multiple antenna systems have the potential to allow multiple users to simultaneously use the same frequency band, making efficient use of r.f. bandwidth. In order to predict the performance of these combining algorithms, knowledge of both the time and angle of each arrival in a multipath environment is needed.

Studies on the distribution of angles of arrival for indoor channels have not been published until very recently. Lo and Litva [4] found that multipath arrivals tend to occur at varying angles indoors, but were not able to arrive at any conclusions based on their limited data. Guerin [5] used a data acquisition system similar to the one used in this present study to collect narrowband angle of arrival data and wideband time arrival data, but they did not collect any data in which the two were measured simultaneously. Wang, et al [6], used a rectangular antenna array to estimate both the elevation and azimuth angles of arrival for major multipaths, but did not measure the corresponding time of arrival. Litva, et al, [7] used a rectangular array to take simultaneous measurements of time and angle of arrival, similar to the format of the data presented here. They came to the preliminary conclusion that it is possible to make accurate measurements of this type and learn more about what is happening in the indoor multipath channel. However, their experiment was not extensive enough to make any conclusions about the channel.

This paper expands on some of this recent research. A data acquisition system is described which was able to collect simultaneous measurements of both time and angle of multipath arrivals over short transmission distances. This enables a joint temporal-spatial characterization of the indoor channel response. Some of the results will be presented, along with general observations about the patterns that were noticed.

\section{Data Acquisition System}

The key to the data acquisition system was that the propagation distances in question were relatively short. This allowed for the possibility of the transmitter and the 
receiver being connected by cable. The actual measurement of the channel temporal response was done using a Hewlett Packard 8720B network analyzer. The output port of the network analyzer was amplified (using a Hewlett Packard $8449 \mathrm{~A}$ preamplifier) and sent to the transmitting antenna (an omnidirectional monopole) via a cable $36 \mathrm{~m}$ in length. This cable was a very low loss, semi-rigid coaxial cable with physical dimensions and electrical characteristics similar to that of RG-8. The transmitted signal was amplified at the antenna by a second amplifier (Miteq AFS4-02001800-35-10P-4). We experienced about $18 \mathrm{~dB}$ of loss over the length of the cable at the $7 \mathrm{GHz}$ frequency chosen for the measurements. The signal was received using a $60 \mathrm{~cm}$ parabolic dish antenna which had a null to null beamwidth of approximately $10^{\circ}$. The signal from the antenna was sent into the input port of the network analyzer. The time domain impulse response of the channel was measured by sampling the transmission coefficient of the channel over a wide band of frequencies using a linear f.m. sweep. An inverse Fourier transform was then used to convert the frequency domain data to the time domain, and to thus yield high resolution channel impulse response measurements. Using this approach, the time of each arrival received at that particular angle could be measured with very low noise levels. All of this processing was done internally to the network analyzer.

In order to measure the time response over all angles, the described impulse responses were collected repeatedly and the narrowbeam antenna rotated at $2^{\circ}$ increments until they had been collected over a $360^{\circ}$ range. The antenna positioner and network analyzer were controlled by a single PC, which downloaded each impulse response as it was calculated. The 180 angular measurements required to generate one complete data set took about 20 minutes to complete. The time of arrival was measured over a window of $200 \mathrm{~ns}$ from 150 to $350 \mathrm{~ns}$ in delay. The $150 \mathrm{~ns}$ delay in window start-up was chosen to account for the bulk twoway time delay down the transmit cable and back through the wireless channel. The sweeps were centered at $7 \mathrm{GHz}$, with a bandwidth of $500 \mathrm{MHz}$. Each impulse response was 401 points in length, providing a time resolution of $1 / 2$ ns. The equivalent temporal pulsewidth after processing of the impulse responses was $3 \mathrm{~ns}$, enabling resolution of distinct multipath arrivals separated by as little as 3ns. An illustration of the system is shown in Fig. 1.

\section{A. Design Considerations}

The frequency of $7 \mathrm{GHz}$ was chosen primarily because of availability of equipment. The assumption was also made that bands higher than the currently available $900 \mathrm{MHz}$ and $2.4 \mathrm{GHz}$ bands will eventually be available for unlicensed indoor applications. Very recently, this has proven to be correct, with new bands in the $5-6 \mathrm{GHz}$ range being allocated by the FCC precisely for this purpose. These higher frequencies have, up to this point, received relatively little attention. However, some recent research has examined the indoor applications of frequencies as high as
$60 \mathrm{GHz}[6]$.

The 6.75 to $7.25 \mathrm{GHz}$ band that was used for data collection was ideal for this research because X-Band equipment was the most readily available, and it requires a smaller aperture to achieve a good angular resolution on the receiving antenna. The center frequency was chosen as low as our X-Band antenna would accommodate, in order to minimize the loss in the long coaxial cable. This cable becomes increasingly lossy with higher frequency. Because the band centered at $7 \mathrm{GHz}$ has not been previously used for indoor measurements, we feel that these experiments can be very useful to provide a general idea of what is happening in the indoor multipath environment at the newly allocated higher frequencies.

It should be noted that the circular aperture of the antenna produces a $6^{\circ} 3 \mathrm{~dB}$ beam width in both the horizontal and vertical directions. This implies that multipath arrivals whose angles of arrival in the vertical plane are significant (i.e., reflections from the ceiling), can be missed by the receiving antenna. In order to determine whether this would affect the results, the receiving antenna was experimentally modified. By masking the antenna, the beam pattern was widened in the vertical plane to approximately $30^{\circ}$ beamwidth, allowing the system to see nearly all major multipaths. Comparison of the data from the system with and without modification to the antenna showed that while some multipath arrivals became more or less visible than others, the overall statistical structure of the multipath environment, including time vs. Angle of arrival, was unchanged. As a result, the antenna was left unmodified for the data collection because of its higher gain in the original configuration.

\section{B. Data Processing}

The data collected by the testbed acquisition system consists of a series of time domain impulse responses of the channel, measured for each test angle. When this data is presented in matrix form, it represents a complete "image" of the channel, such as the one shown in Fig. 2.

The multipath channel is assumed to be entirely specular, which implies that the image plots can be modeled as a collection of point sources blurred by a point spread function (due to a non-ideal antenna beampattern and the network analyzer response) and corrupted by additive noise. This assumption reduces the problem of identifying exact time and angle of arrival to a simplified deconvolution. The exact point source times and angles were estimated using the CLEAN algorithm, which was originally used for processing of astronomical images, also often modeled as point sources convolved with a blurring function. The algorithm is a recursive subtraction of the point spread function from the image, with the point spread function positioned to correspond with the maximum value of the image. The highest peak is found, its amplitude, time, and angle are stored, and a scaled copy of the impulse response is subtracted from the image. This process is repeated on the residual image until a threshold is reached. The result 
of this application of the CLEAN algorithm to the data of Fig. 2 is shown in Fig. 3.

Early analysis of the data showed clustering effects in both time and angle, as will be discussed in section III. An attempt was made to categorize the arrivals into clusters using a computerized algorithm, but this proved to be very difficult. Since the clusters were obvious to the observer in most cases, and due to the small amount of data overall, the clusters were picked by hand. Knowing the time, angle, and amplitude of each multipath arrival gives sufficient information to analyze the statistics of the indoor multipath channel.

\section{RESULTS}

A total of 65 data sets were collected in two buildings on the campus of Brigham Young University. The first 55 data sets were collected on the fourth floor of the Clyde Building, which is constructed of reinforced concrete and cinder block, with all internal walls being cinder block. For comparison, 10 additional data sets were collected in the nearby Crabtree building, which is constructed with steel girders, and with internal walls of gypsum board over a steel frame. In all of the data that was collected, the transmitter and receiver had some kind of wall between them, and in most cases they were in different rooms. Separation distances ranged from approximately 20 feet to 100 feet. Most doors in both buildings were wood, and were closed for all measurements.

\section{A. General Observations}

Observing the raw data, it is apparent that the multipath channel is dominated by specular multipath. This is supported by the fact that multipath arrivals appear highly localized in time and angle. The arrivals due to multipath can be assumed to be reflections from walls, large furniture, and smaller objects which are highly conductive. Diffuse multipath in an outdoor environment is generally due to reflective surfaces that are not smooth, such as foliage. Man-made objects, which almost wholly comprise the indoor environment, tend to be smooth, especially at microwave frequencies. Diffuse multipath may exist indoors, but it appears that its presence is negligible in this data. This validates the assumption of point-like sources in the time delay-angle plots, and justifies using the CLEAN algorithm for processing the data. This will only work if specular multipath dominates.

A clustering pattern in both time and angle was immediately visible in the data images. Generally there were at least two or three clusters, except in rare cases with long propagation distances and consequently low signal to noise ratios. There were some extreme cases of more than five clusters, especially in the Crabtree Building data.

In both buildings, the line of sight path was generally observable in the data, but its attenuation was dependent on the number and type of walls between the transmitter and receiver. This direct path almost always corresponded to the dominant cluster. Weaker clusters generally cor- responded to back wall reflections and openings such as doorways, even though all doors were closed during data collection.

In general, greater transmitter/receiver separation corresponded to fewer clusters and lower signal to noise ratios. Higher numbers of clusters tended to be the results of a stronger signal, and more complex geometry of the channel, i.e. more doorways, walls, or rooms in between the transmitter and receiver.

The clusters tended to occur at any angle. For most clusters, the majority of elements were contained within 20 to 30 degrees of the cluster mean, with occasional outlying elements. Clusters associated with doorways had lower angular variance than usual.

\section{B. Clyde Building}

Fig. 4 is representative example of a Clyde Building raw data set. There are 4 very obvious clusters. The strongest arrivals in each cluster are those that arrive earliest in time. The amplitudes of the arrivals in each cluster appear to decay over time until they disappear into the noise floor. It is also clear from the data that the arrivals in each cluster tend to be grouped closely in angle.

Fig. 5 is an example of a data set with only two clusters. This set was collected in a scenario where the transmitter and receiver were in neighboring rooms, with a single wall and a short distance separating them. The overall power is noticeably higher in this case, and the two clusters have many more visible arrivals. The stronger of the two clusters can be attributed to the direct path through the wall, and the weaker one to back wall reflections. Although not visible in this case, it was found that doorways often cause at least one cluster, depending upon the particular scenario.

\section{Crabtree Building}

The Crabtree Building data showed similar characteristics, but with some important differences. A plot typical of the data from the Crabtree Building is shown in Fig. 6 . There were on average more clusters per location in the Crabtree building, with at least 7 identifiable clusters in this case. The angular clustering was quite similar, but the length in time of the average cluster was considerably longer, since the decay over time of the arrivals in a cluster appeared to be much slower. It was also noted that clusters were much stronger in amplitude than clusters received at a similar transmission distance in the Clyde Building. This indicates that the steel and gypsum board construction tends to be more reflective due to the presence of the steel, and attenuates less due to the gaps which are covered with gypsum board. This also explains the higher overall number of clusters seen in the Crabtree Building.

\section{Comparison}

In the Crabtree Building, the line of sight through walls was stronger than in the Clyde Building. However, in the Clyde Building, the line of sight path still tended to be 
very strong. Both the Clyde and Crabtree Building data exhibited similar clustering structures. There were some differences that reflect the properties of the different types of building materials. The Crabtree Building data produces a higher total number of arrivals per data set, and a slightly higher number of clusters. In the Crabtree Building the average number of clusters per data set was about 5 , and in the Clyde Building the average was 3.

There is a marked difference between the buildings in the attenuation of arrivals with increasing delay. The clusters appear to decay much more slowly and are consequently longer in time-spread-of-arrival in the Crabtree Building as compared to the Clyde Building. There also appears to be a lower variance in angle within clusters in the Crabtree Building.

\section{Conclusion}

The indoor multipath channel appears to behave at 7 $\mathrm{GHz}$ very similarly to that which was observed by Saleh and Valenzuela [1] at lower frequencies. Specifically, arrivals tend to come in clusters, with the strongest clusters arriving first, and the strongest arrivals in each cluster also arriving first. As the delay time increases, the power of the clusters and the arrivals within the clusters tend to decay in amplitude until they disappear into the noise floor.

There appears to be a strong clustering effect in angle, where arrivals that are in the same cluster tend to be close in angle. The clusters themselves tend to come at any angle. It can be concluded that in general the angle of arrival in an indoor multipath environment is not uniformly distributed.

Comparison of the $7 \mathrm{GHz}$ data with other researchers' data at lower frequencies leads to the conclusion that the qualitative multipath behavior is similar over a wide range of frequencies, although the qualitative aspects such as attenuation and the statistical properties of the clustering patterns are probably more frequency dependent. We noted a significant dependence of the detailed arrival distribution properties on the type of building construction. This suggests that collecting data from a wide range of building types would be needed for useful indoor wireless performance predictions. The data gathering system presented here is a useful setup that can be adapted for a variety of other frequencies for collection of other data. In addition to collection of more data, more detailed statistical analyses, such as the one presented in [3], are important and suggested as areas of further research into understanding indoor multipath propagation.

\section{REFERENCES}

[1] Adel A. M. Saleh and Reinaldo A. Valenzuela. A statistical model for indoor multipath propagation. IEEE Journal on Selected Areas of Communications, SAC-5:128-13, February 1987.

[2] George L. Turin et al. A statistical model of urban multipath propagation. IEEE Transactions on Vehicular Technology, VT21(1):1-9, February 1972.

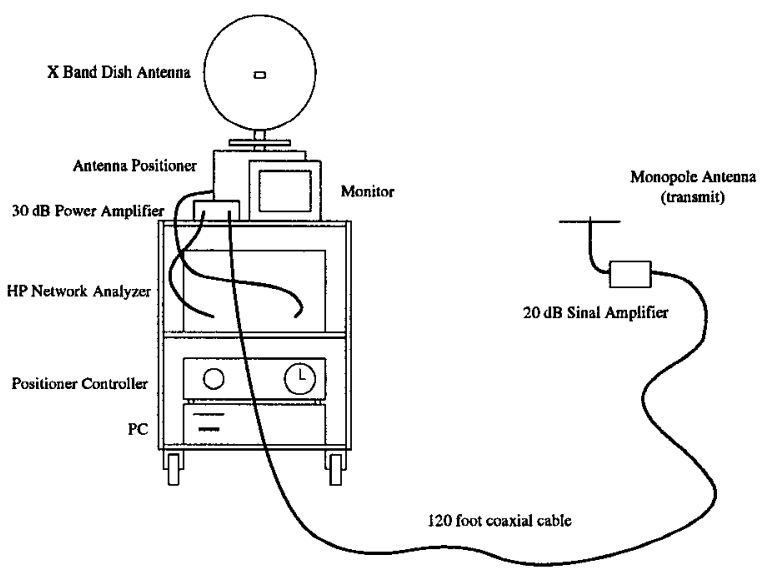

Fig. 1. The Data Acquisition System

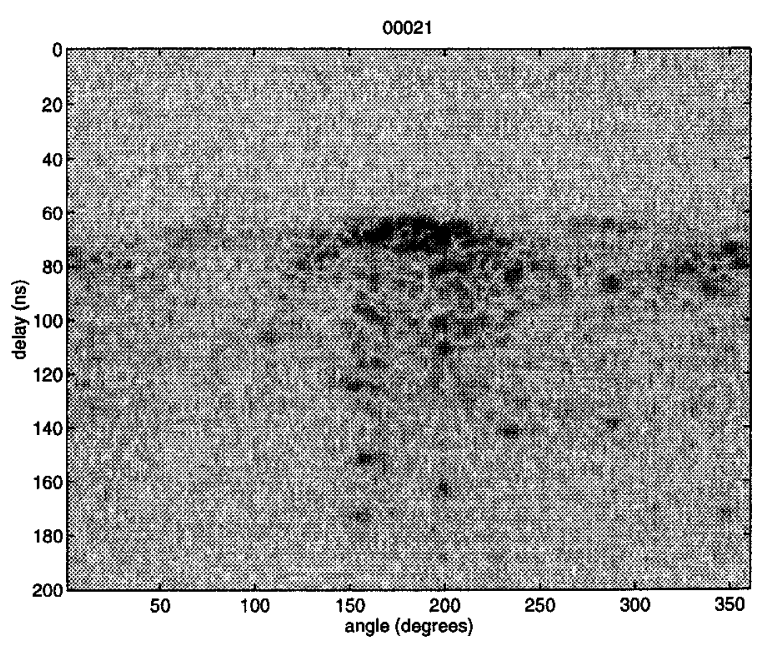

Fig. 2. A typical raw data set

[3] Quentin Spencer, Michael Rice, Brian Jeffs, and Michael Jensen. A statistical model for angle of arrival in indoor multipath propagation. In IEEE Vehicular Technology Conference. IEEE, 1997.

[4] T. Lo and J. Litva. Angles of arrival of indoor multipath. Electronics Letters, 28(18):1687-1689, August 271992.

[5] Stephane Guerin. Indoor wideband and narrowband propagation measurements around $60.5 \mathrm{ghz}$. in an empty and furnished room. In IEEE Vehicular Technology Conference, pages 160$164,1996$.

[6] Jian-Guo Wang, Ananda S. Mohan, and Tim A Aubrey. Anglesof-arrival of multipath signals in indoor environments. In IEEE Vehicular Technology Conference, pages 155-159. IEEE, 1996.

[7] John Litva, Amir Ghaforian, and Vytas Kezys. High-resolution measurements of aoa and time-delay for characterizing indoor propagation environments. In IEEE Antennas and Propagation Society International Symposium 1996 Digest, volume 2, pages 1490-1493. IEEE, 1996. 


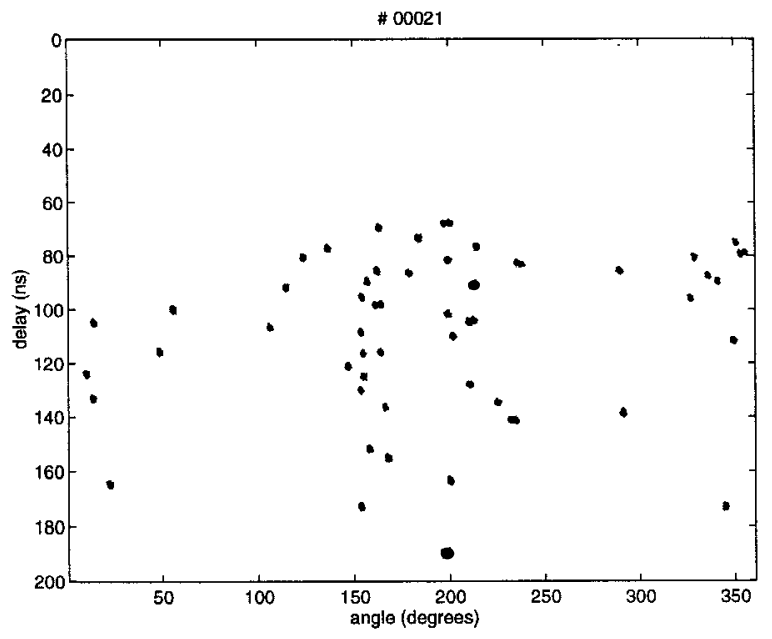

Fig. 3. The data set in Fig. 2 after processing
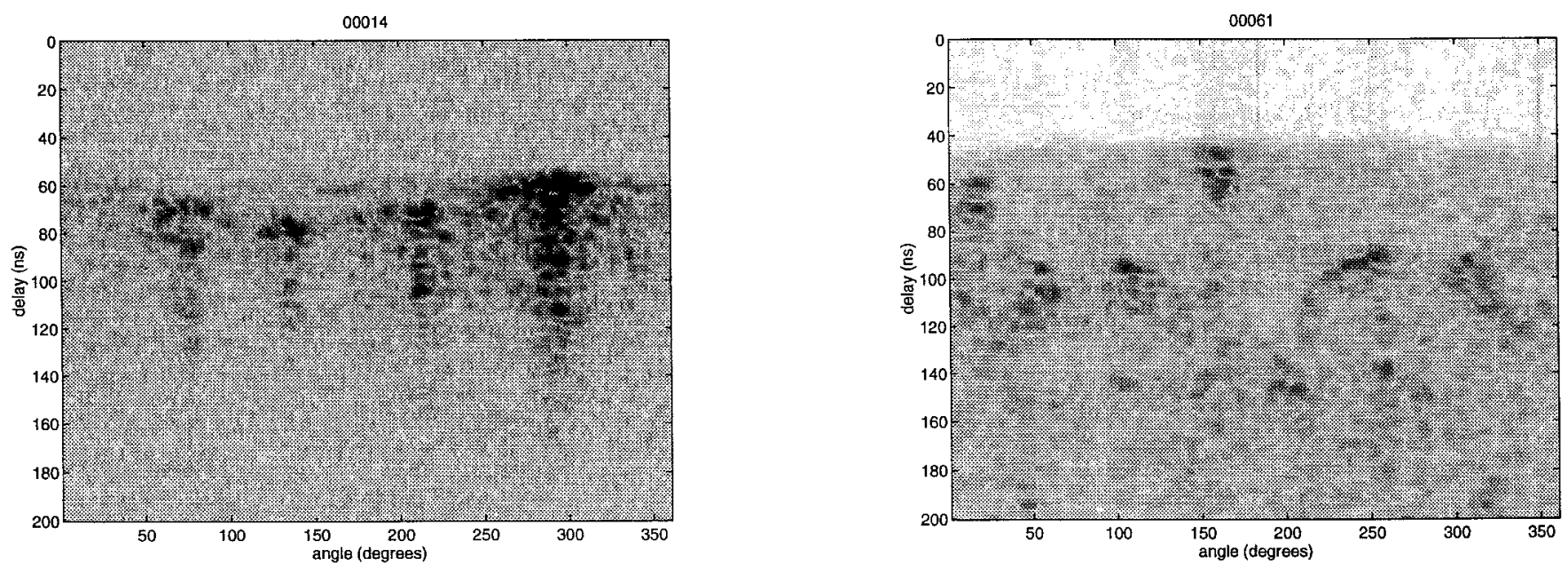

Fig. 4. Plot \#14

Fig. 6. Plot \#61

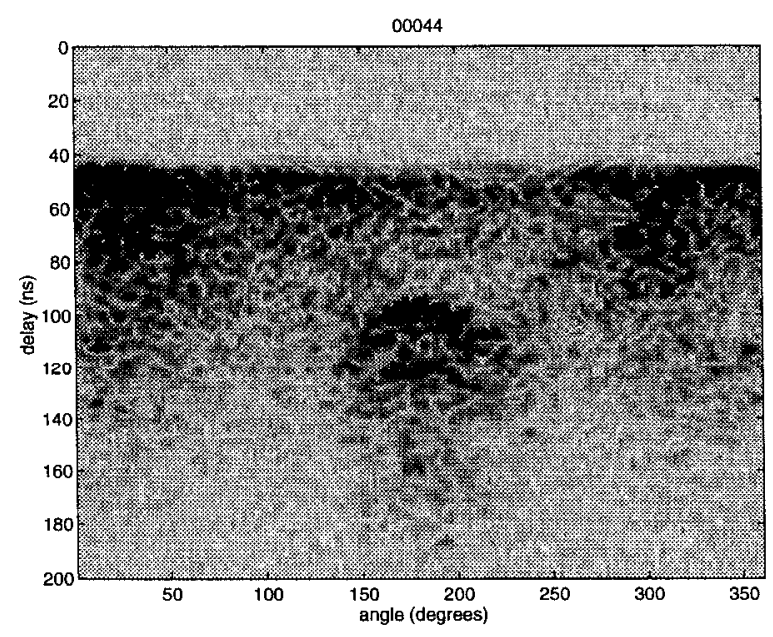

Fig. 5. Plot \#44 